\title{
Nanoscale properties of GEMS-like material in primitive carbonaceous chondrites
}

\author{
K. L. VILLALON ${ }^{123, s}$, K. K. OHTAKI ${ }^{4}$, J. P. BRADLEY,
} H. A. ISHII ${ }^{4}$, P. R. HECK ${ }^{123}$, K. KEATING ${ }^{123}$, A. M. DAVIS ${ }^{123}$, AND T. STEPHAN ${ }^{123}$

Department of the Geophysical Sciences, The University of Chicago, Chicago, IL, USA

${ }^{2}$ Chicago Center for Cosmochemistry

${ }^{3}$ Robert A. Pritzker Center for Meteoritics and Polar Studies, Field Museum of Natural History, Chicago, IL, USA

'Hawai'i Institute of Geophysics \& Planetology, University of Hawai' $i$ at Mānoa, Honolulu, HI, USA

(*correspondence: kvillalon@uchicago.edu)

A major component of primitive carbonaceous chondrite (CC) matrices is amorphous silicate, a phase that is highly susceptible to alteration and whose presence is therefore indicative of minimal secondary processing. Amorphous silicate is also a dominant component of the interstellar medium (ISM) as well as chondritic porous interplanetary dust particles (CP-IDPs), a subset of highly porous IDPs believed to originate in comets and considered to be amongst the most pristine extraterrestrial samples found to date.

Amorphous silicates with embedded nano-inclusions have been reported in rare primitive $\mathrm{CCs}[1,2,3]$. They have morphological similarities as well as comparable average chemical compositions to glass with embedded metal and sulfides (GEMS) found in CP-IDPs and micrometeorites believed to originate from comets.

Using STEM nanodiffraction with (conventional) TEM and STEM EDS, we have determined the mineralogy, crystallography, and elemental compositions of the inclusions embedded in the GEMS-like objects of the Paris CM chondrite. Contrary to bona fide GEMS in IDPs, we have not identified any metal inclusions embedded in the amorphous silicate matrix of Paris, but we have identified rare nm-sized metal grains that reside outside of the amorphous silicate domains. GEMS-like material in Paris differs in morphology, composition, and mineralogy from IDP GEMS at the nanoscale. We have also identified GEMS-like material in the matrix of the recent fall Aguas Zarcas (CM) [4]. This material more closely resembles the GEMS-like material in Paris and in other primitive CCs than in IDP GEMS.

[1] Leroux H. et al. (2015) GCA, 170, 247-265. [2] Keller L. P. et al. (2009) MAPS, 44, \#5371. [3] Nittler L. R. et al. (2019) Nat. Astron., 3, 659-666. [4] Villalon K. L. et al. (2020) LPS, 51 , \#2757. 